\title{
CYP2C 19 and nongenetic factors predict poor responsiveness to clopidogrel loading dose after coronary stent implantation
}

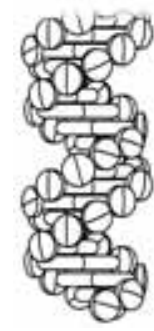

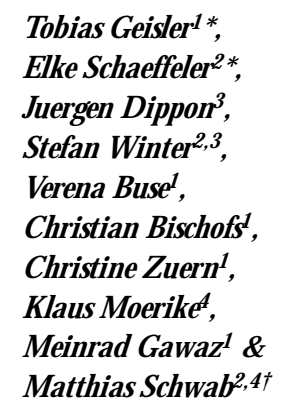

${ }^{\dagger}$ Author for correspondence

${ }^{1} D$ epartment of Cardiology, University $\mathrm{H}$ ospital

Tuebingen, O tfried-M uellerStrasse 10, Tuebingen,

Germany

Tel.: +49 70712983688 ;

Fax: +49 707129 5749;

E-mail: meinrad.gawaz@ med.uni-tuebingen.de

${ }^{2} \mathrm{D} \mathrm{r} \mathrm{M}$ argarete-Fischer-Bosch Institute of Clinical

Pharmacology, Stuttgart, and U niversity of Tuebingen,

Auerbachstrasse 112

Germany

Tel.: +49 7118101 3700;

Fax: +49 711859 295;

E-mail: matthias.schwab@

ikp-stuttgart.de

${ }^{3} \mathrm{D}$ epartment of $\mathrm{M}$ athematics, U niversity of Stuttgart,

Stuttgart, Germany

${ }^{4} \mathrm{D}$ epartment of Clinical

Pharmacology, U niversity

H ospital Tuebingen,

Tuebingen, Germany

*Both authors contributed equally to this work

\footnotetext{
Keywords: clopidogrel, coronary artery disease, cytochrome P450 2C 19, percutaneous coronary intervention, pharmacogenetics, PREDICT score

\begin{abstract}
Aims: To investigate an association of responsiveness to clopidogrel loading dose with genotypes of cytochrome P450 (CYP) 2C19, other CYP isozymes and nongenetic factors in patients with coronary artery disease. Materials \& methods: Genotyping for CYP2C19 $(* 2, * 3$ and $* 17)$, CYP3A $4 * 1$ B and CYP3A $5 * 3$ variants was performed in patients $(n=237)$ who underwent percutaneous coronary intervention. Adenosine diphosphate-induced platelet aggregation was determined after first administration of $600 \mathrm{mg}$ clopidogrel. Results: CYP2C19*2 carriers showed significantly increased residual platelet aggregation (RPA) (OR: 4.6; 95\% Cl: 2.5-8.7; $p$ <0.0001) compared with noncarriers. All other polymorphisms had no influence on RPA. For the development of a risk score for better prediction of RPA, CYP2C19*2 genotype and previously identified nongenetic risk factors (age $>65$ years, Type 2 diabetes mellitus, decreased left ventricular function, renal failure and acute coronary syndrome) were analyzed. Multivariable logistic regression analysis showed a significant correlation of the nongenetic factors $\left(\chi^{2}=5.32 ; p=0.021\right)$ and CYP2C19*2 $\left(\chi^{2}=21.31 ; p<0.0001\right)$ with high RPA, and an even higher association for the combination of both $\left(\chi^{2}=25.85 ; p<0.0001\right)$. Conclusions: Prediction of responsiveness after clopidogrel loading dose may substantially be improved by adding CYP2C19*2 genotype to nongenetic risk factors.
\end{abstract}

Intracoronary stent implantation has gained substantial success in the past owing to technological and therapeutic advances. Pretreatment with a loading dose of clopidogrel, an inhibitor of platelet aggregation induced by ADP, was shown to significantly improve clinical outcome in patients undergoing percutaneous coronary intervention (PCI) [1,2]. However, marked interindividual variability in clopidogrel-induced inhibition of platelet aggregation is well-known and up to $30 \%$ of patients do not achieve an adequate antiplatelet effect after an initial clopidogrel loading dose [3-7]. In a large-scale study of 1092 consecutively enrolled patients, we recently identified nongenetic factors (age $>65$ years, Type 2 diabetes mellitus, decreased left ventricular function, renal failure and acute coronary syndrome) for better prediction of patients with persistent high residual platelet activity after coronary stenting and dual antiplatelet therapy with clopidogrel and aspirin [8]. U sing a factor-weighted model, a score (termed residual platelet aggregation after deployment of intracoronary stent [PREDICT ]-score) at least higher than 4 score levels significantly increased the risk for persistent residual platelet aggregation (RPA). Of note, preliminary data indicate that after short-term follow-up of 30 days, the incidence of major adverse cardiovascular events (e.g., myocardial infarction, cardiovascular death and ischemic stroke) was significantly increased in patients with high RPA [8].

In addition to nongenetic factors, putative mechanistic explanations of elevated RPA include functional polymorphisms in enzymes involved in the metabolism of clopidogrel. In favor, major evidence came from a healthy volunteer study demonstrating that carriage of the cytochrome P4502C 19 loss of function variant CYP2C19*2 has been associated with markedly decreased platelet responsiveness to clopidogrel [9]. Exertion of the antiplatelet effect of clopidogrel requires in vivo conversion to an active metabolite, an unstable thiol compound, inhibiting platelet aggregation by the formation of a disulfide bridge between the thiol group of the active compound and a cysteine residue of the $P_{2} Y_{12}$ receptor of platelets [10]. In addition to CYP2C 19, the cytochrome P450 enzyme family CYP3A substantially contributes to the bioactivation of clopidogrel. In vitro and in vivo data conclusively demonstrate that CYP3A4 is also involved [11,12].

Therefore, the present study was conducted to investigate comprehensively the contribution of functional polymorphisms in the drug-metabolizing enzymes, CYP2C 19 and CYP3A4/5, on 
clopidogrel responsiveness, in addition to nongenetic factors in patients undergoing coronary intervention for symptomatic coronary artery disease (CAD).

\section{Methods}

Study population \& design

From July 2006 to M arch 2007, patients admitted to the $D$ epartment of C ardiology, U niversity Hospital Tuebingen, Germany, for coronary intervention for symptomatic CAD were consecutively enrolled in this pharmacogenetic trial. $O$ nly patients of $C$ aucasian origin were included. M oreover, 186 healthy unrelated individuals of Caucasian (German) origin, randomly recruited as described previously [13], were used as an independent control population to compare CYP allele frequencies between patients and controls. The study protocol was approved by the ethics committee of the M edical Faculty of the U niversity of Tuebingen, G ermany, and all patients and healthy controls gave their written informed consent to participate.

The study protocol specified the following criteria:

- Only patients with symptomatic CAD (stable angina or acute coronary syndrome) were included;

- Patients with known disorders of platelet function or treatment with glycoprotein $\mathrm{IIb} / \mathrm{ll}$ a receptor inhibitors 1 week prior to inclusion were excluded;

- A loading dose of $600 \mathrm{mg}$ clopidogrel was given prior to $\mathrm{PCl}$ followed by a daily dose of 75 mg clopidogrel;

- All patients received $500 \mathrm{mg}$ aspirin intravenous before $\mathrm{PCl}$, followed by oral aspirin $100 \mathrm{mg}$ per day, unless aspirin was contraindicated owing to gastrointestinal bleeding or allergic reactions;

- Unfractionated heparin (70 units per kg body weight intravenously) was administered to all patients prior to $\mathrm{PCl}$.

Acute coronary syndrome was diagnosed if at least one of the following criteria was met: unstable angina (clinical symptoms and ECG changes without evidence of myocardial necrosis), acute myocardial infarction (positive markers of myocardial necrosis, for example, elevation of serum troponin and creatine kinase-myoglobin binding [CK-MB]) including ST-segment elevation myocardial infarction (STEM I), and non-STEM I (N STEM I).
On the basis of our previous work including 1092 patients, we defined poor response to clopidogrel loading dose if the level of the individual RPA measured at least $6 \mathrm{~h}$ after first administra tion belong to the upper tertile of RPA levels with a predefined cut-off value of $47 \%$ [8].

Blood sampling \& platelet aggregation Patient blood was collected in $3.8 \%$ citrate plasma at least $6 \mathrm{~h}$ after first administration of $600 \mathrm{mg}$ clopidogrel, when maximum platelet inhibition is achieved [14]. In brief, samples were centrifuged at $1000 \mathrm{rpm}$ for 10 min to obtain platelet-rich plasma (PRP) and additionally 10 $\mathrm{min}$ at $3500 \mathrm{rpm}$ to recover platelet-poor plasma (PPP). The platelet concentration of PRP was adjusted to $2 \times 10^{5} / \mu \mathrm{l}$ by adding homologous PPP. Percent platelet aggregation after stimulation with $20 \mu \mathrm{mol} / \mathrm{I}$ AD P was assessed with the turbodimetric method using a Chronolog Lumi aggregometer with Aggro-Link Software as described previously [15]. RPA was defined as aggregation measured $5 \mathrm{~min}$ after addition of AD P $20 \mu \mathrm{mol} / \mathrm{l}$. We decided to use final platelet aggregation because it reflects stabilization of aggregates mediated by the $\mathrm{P}_{2} \mathrm{Y}_{12}$ AD $\mathrm{P}$ receptor, which is blocked by clopidogrel, whereas peak platelet aggregation rather reflects $\mathrm{P}_{2} \mathrm{Y}_{12}$-mediated platelet shape change independently from clopidogrel effects [16].

\section{Genotyping of CYP450 variants}

Genomic DNA was isolated from ethylenediaminetetraacetic acid (EDTA) blood samples using the Q IAmp ${ }^{\circledR}$ DNA Blood M ini Kit System (Q iagen, Hilden, Germany). Candidate genes of cytochrome P450 enzymes were selected on the basis of metabolic pathways known to be involved in clopidogrel metabolism $[9,11,12]$. The criteria for selection of variants in these genes were a representative allele frequency in Caucasians and clear evidence for functional consequences based on in vitro/in vivo data [101]. Thus, the following polymorphisms of CYP2C19 were analyzed: CYP2C 19*2 (681G >A), *3 (636G >A) and $* 17 \quad(-806 C>T)$, as well as the variants CYP $3 A 4 * 1 B \quad(-392 A>G)$ and CYP3A5 *3 $(6986 A>G)$ in other CYP enzymes. Genotyping for CYP2C 19 variants $* 2, * 3$ and $* 17$, as well as CYP $3 A 4 * 1 B$ and CYP $3 A 5 * 3$, was performed by matrix-assisted laser desorption/ionization time-of-flight mass spectrometry (M ALD ITOF M S) using the M assARRAY ${ }^{\circledR}$ Compact system (Sequenom, CA, USA) as previously 
described [17]. D etails of primers and assays are available upon request. Approximately $10 \%$ of samples within each assay were retyped as a quality control. Laboratory staff were blinded to the case status of the study participants during the entire genotyping process.

Statistical analysis

To assess the power of the study we note that the frequency of the CYP2C19*2 allele is approximately $15 \%$ in healthy Caucasians, which amounts to observing approximately $28 \%$ persons carrying the allele. D ue to the definition of high RPA, it is expected that approximately $30 \%$ of the patients not carrying the allele show high RPA. To detect an odds ratio (OR) of at least 2.5 describing the association of RPA and genotype, 230 patients have to be included in the study to achieve a power of $86 \%$.

All values are presented as median and interquartile range. Univariate analyses were performed to identify genotypes of major influence on responsiveness to clopidogrel. $\chi^{2}$ analysis or Fisher's exact test were used as appropriate.

The dichotomous logistic regression model was computed using the statistics software R, version 2.4.1 [102] and the Design library [18]. OR are presented with $95 \%$ confidence intervals $(\mathrm{Cls})$ and two-sided $\mathrm{p}$-values. Statistical significance was defined as $p<0.05$.

0 bserved and expected allele and genotype frequencies within populations were compared by means of $\mathrm{H}$ ardy-Weinberg equilibrium calculations [103].

Results

Demographic \& dinical data

Base line characteristics of the 237 consecutively enrolled study patients treated with clopidogrel after implantation of stents owing to symptomatic CAD are summarized in Table 1. A median RPA of $34 \%$ (interquartile range $33.8 \%$ ) was found. According to our previous large-scale study [8], we used the predefined RPA cut-off value of $47 \%$ to categorize all 237 patients into subjects with a lower ( $n=175$, responder) or higher ( $n=62$, poor responder) RPA. In the majority of patients, RPA was determined at around $20 \mathrm{~h}$ (median: $21 \mathrm{~h} \mathrm{[5.1-285.0])}$ after loading dose of $600 \mathrm{mg}$. Time difference in the two groups with low (median: $21 \mathrm{~h}$ [5.1-285.0]) and high (median: $22 \mathrm{~h}$ [5.9-177.0]) RPA was not significantly different. A total of 106 $(44.7 \%)$ patients were treated for acute coronary syndrome; $35 \%$ of patients suffered from Type 2 diabetes. In the present study, the frequency distribution of all clinical parameters (Table 1), except hyperlipidemia, was similar to our previous results, which included 1092 individuals [8]. In analogy to these data, we defined the individual PREDICT score based on the five nongenetic risk factors (age $>65$ years, acute or stable coronary syndrome, Type 2 diabetes, decreased left ventricular function and renal failure) for all 237 patients. As expected, the use of comedications was frequent and predominately statins $(66 \%)$, angiotensin-converting enzyme (ACE) inhibitors (69\%), AT 1 receptor antagonists $(12 \%), \quad \beta$-blockers $(79 \%)$ and calcium antagonists (19\%) were prescribed.

Association with CYP polymorphisms 0 verall, no significant differences between allele and genotype frequencies for all tested CYP polymorphisms were found when patients and healthy volunteers were compared (Table 2). As expected, owing to the rare allele frequency, no carrier of CYP2C 19*3 was detected. All genotype frequencies of CYP polymorphisms in healthy controls were in $\mathrm{H}$ ardy-Weinberg equilibrium. Again this was the case in the patient group of responders as well as poor responders except CYP2C19*17 (only in nonresponders), which may be explained by the low sample size. Carriers of the CYP2C19*2 variant showed significantly increased RPA after clopidogrel loading dose compared with noncarriers (Figure 1) and the median RPA of heterozygous (46\%) and homozygous carriers (54\%) was considerably higher than in noncarriers (30\%). RPA differed significantly between the three CYP2C19*2 genotype groups when patients were categorized into responders (Iow RPA) and poor responders (high RPA) $\left(\chi^{2}=27 ; p<0.001\right)$. These data hold true when patients who were heterozygous or homozygous for variant CYP 2 C $19 * 2$ were pooled together (e.g., CYP2C19*1/*1 vs CYP 2 C $19 * 1 / * 2$ plus $* 2 / * 2 ;$ OR: $4.6 ; 95 \% \mathrm{Cl}$ : $2.5-8.7 ; p<0.0001)$. Carriers of at least one CYP2C19*2 allele had a 4.38 -fold increased risk ( $p<0.0001 ; 95 \% \mathrm{Cl} 2.3-8.33$ ) for developing high RPA (>47\%). All other polymorphisms tested (CYP2C19*17, CYP3A4*1B and CYP3A5*3) had no influence on RPA (Table 3), with or without pooling heterozygote and homozygote variant patients. Moreover, the median RPA of heterozygous (30\%) and homozygous carriers (37\%) of CYP 2 C $19 * 17$ were not significantly different compared with noncarriers $(36.0 \%)$. 
Table 1. Clinical characteristics of patients according to their individual residual platelet activity.

\begin{tabular}{|c|c|c|c|}
\hline Variables & Low RPA ( $n=175)$ & High RPA $(n=62)$ & Total $(n=237)$ \\
\hline Residual platelet activity,\%* & $26.0(27.0)$ & $54.00(9.0)$ & $34.00(33.8)$ \\
\hline Age (years)* & $69.0(13.0)$ & $69.0(11.3)$ & $69.0(13.0)$ \\
\hline \multicolumn{4}{|l|}{ Sex, no. (\%) } \\
\hline Male & $136(77.7)$ & $45(72.6)$ & $181(76.4)$ \\
\hline Female & $39(22.3)$ & $17(27.4)$ & $56(23.6)$ \\
\hline Acute coronary syndrome, no. (\%) & $76(43.4)$ & $30(48.4)$ & $106(44.7)$ \\
\hline STEMI & $21(12.0)$ & $9(14.5)$ & $30(12.7)$ \\
\hline NSTEM I & $40(22.9)$ & $14(22.6)$ & $54(22.8)$ \\
\hline \multicolumn{4}{|c|}{ Left ventricular function (ejection fraction), no. (\%) } \\
\hline $45-55 \%$ & $45(25.7)$ & $17(27.9)$ & $62(26.3)$ \\
\hline $35-45 \%$ & $14(8.0)$ & $12(19.7)$ & $26(11.0)$ \\
\hline$<35 \%$ & $23(13.1)$ & $9(14.8)$ & $32(13.6)$ \\
\hline Diabetes, no. (\%) & $56(32.0)$ & $26(41.9)$ & $82(34.6)$ \\
\hline Hypertension, no. (\%) & $147(84.0)$ & $55(88.7)$ & $202(85.2)$ \\
\hline Renal failure, no. (\%) & $20(11.5)$ & $14(22.6)$ & $34(14.4)$ \\
\hline \multicolumn{4}{|l|}{ (serum creatinine $>1.5 \mathrm{mg} / \mathrm{dl}$ ) } \\
\hline Smoking, no. (\%) & $67(38.3)$ & $22(35.5)$ & $89(37.6)$ \\
\hline Hyperlipidemia, no. (\%) & $129(73.7)$ & $46(74.2)$ & $175(73.8)$ \\
\hline
\end{tabular}

${ }^{*}$ Median (interquartile range).

NSTEMI: Non-STEMI: STEMI: ST-segment elevation myocardial infarction; RPA: Residual platelet aggregation.

Multivariable logistic regression analysis In addition to the PREDICT score exclusively based on nongenetic factors, we developed a risk score model including CYP2C19*2 genotypes. The PREDICT score was defined as previously described [8], using a weighting factor of 1-3 for each variable to account for the unequal influence of nongenetic parameters. Thus, age and acute coronary syndrome were weighted by factor 1 , diabetes and renal failure by factor 2 , and left ventricular dysfunction by factor 3 , thus giving scores ranging from 0 to 9 . Of note, multivariable logistic regression analyses showed a significant correlation of the nongenetic risk factors $\left(\chi^{2}=5.32 ; p=0.021\right)$, as well as the

Table 2. Frequency distribution of variant CYP alleles in patients and healthy controls.

\begin{tabular}{lrr} 
& $\begin{array}{r}\text { Patients,\% } \\
(\mathbf{n}=\mathbf{2 3 7})\end{array}$ & $\begin{array}{r}\text { Healthy subjects,\% } \\
(\mathbf{n}=\mathbf{1 8 6})\end{array}$ \\
\hline CYP2C19*2 & $15.2^{\ddagger}$ & $15.2^{\S}$ \\
CYP2C19*3 & 0 & 0 \\
CYP2C19*17 & 25.5 & 25.7 \\
CYP3A4*1B & 3.4 & 4.6 \\
CYP3A5*3 & 92.8 & 88.9 \\
\hline
\end{tabular}

₹CYP2C19 genotypes: *1/*1, $\mathrm{n}=175(74 \%) ; * 1 / * 2, \mathrm{n}=52(22 \%) ; * 2 / 2, \mathrm{n}=10(4 \%)$. ${ }^{\S}$ CYP2C19 genotypes: *1/*1, $\mathrm{n}=135(73 \%) ; * 1 / * 2, \mathrm{n}=42(23 \%) ; * 2 / * 2, \mathrm{n}=7(4 \%)$.
CYP2C19*2 genotype $\left(\chi^{2}=21.31 ; p<0.0001\right)$ with high RPA, but interestingly an even higher association was found for the combination of both $\left(\chi^{2}=25.85 ; p<0.0001\right)$. The risk for developing high RPA increased by the presence of one CYP2C19*2 allele (OR: $3.71 ; 95 \% \mathrm{Cl}$ : 1.87-7.35), and was even more pronounced by the presence of two variant alleles (OR: 10.72; $95 \% \mathrm{Cl}: 2.56-44.88$ ).

On the basis of the logistic regression model we developed a final proportional odds model (nomogram, Figure 2). It allows one to calculate the probability of experiencing a RPA higher than $47 \%$ for a given patient treated with a clopidogrel loading dose of $600 \mathrm{mg}$. In the first step (Figure 2A), for each factor on the left of panel A (i.e., weighted PREDICT score and CYP 2C19*2 genotype [0: wild-type, 1: heterozygous, 2: homozygous variant]) the appropriate number of points is determined on the upper scale and added up. In a second step (Figure 2B), the total sum of points is transformed into the corresponding estimated probability for high RPA $(>47 \%)$, indicating poor response. Thus, the maximal number of points is 165 , predicting a greater than $80 \%$ risk of high RPA. In contrast, the maximal number of points achievable in a patient with a wild-type CYP2C19 is 65 , predicting a risk of approximately $40 \%$. 
Figure 1. Genotype distribution of the CYP2C19*2 polymorphism in patients with high and low residual platelet aggregation receiving a loading dose of $600 \mathrm{mg}$ clopidogrel.

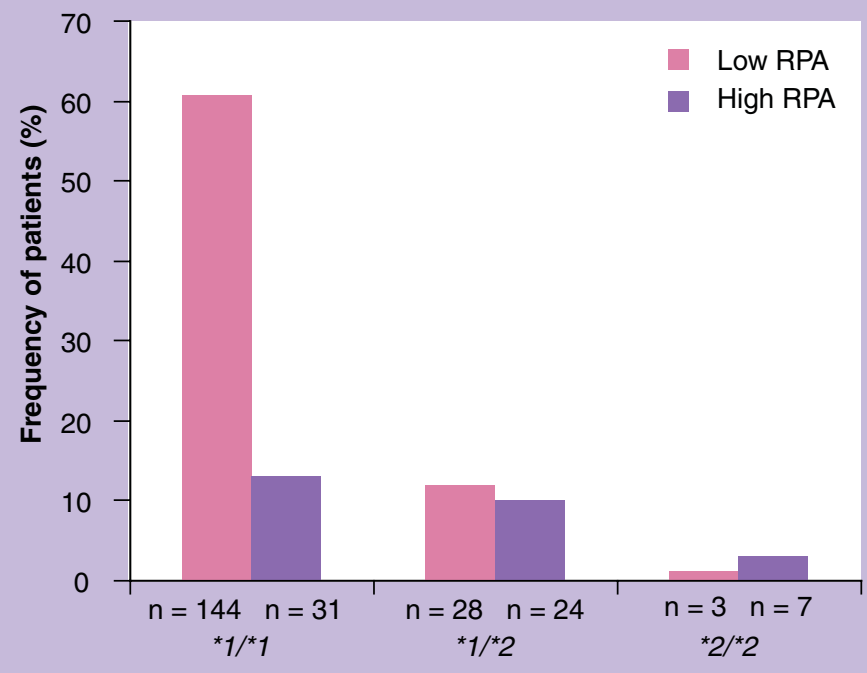

CYP2C19*2 genotype

An increasing incidence of carriers of at least one CYP2C19*2 allele was found in patients with high RPA $(>47 \%)$.

RPA: Residual platelet aggregation. of the vasodilatator phosphoprotein and platelet aggregation profile [9]. This study was later confirmed by the same authors [20], again indicating a significant reduction in AD P-induced platelet aggregation related to CYP2C $19 * 2$ genotype $(p=0.001)$ in a larger cohort of 94 Caucasian medical students treated with a loading dose of $300 \mathrm{mg}$ clopidogrel. M oreover, Brandt et al. [21] who had already claimed in 2006 such an association [22], investigated the contribution of CYP2C19 polymorphisms in 74 healthy subjects predominantly with Caucasian ethnic backgrounds receiving a $300-\mathrm{mg}$ loading dose of clopidogrel. These authors provided first evidence that the presence of CYP2C 19*2 was significantly associated with alteration of pharmacokinetic parameters of the active metabolite of clopidogrel, for example, decreased $A \cup C_{0-24}$, and $C_{\max }$. Very recently, the impact of CYP2C19 genetics on clopidogrel response variability has been investigated in the clinical setting of high-risk vascular patients who underwent primary $\mathrm{PCI}$ and received dual antiplatelet therapy with a loading dose of $600 \mathrm{mg}$ clopidogrel and of $500 \mathrm{mg}$ of aspirin intravenously [23]. In fact, CYP 2C 19*2 genotype distribution differed significantly between patients with and without residual platelet activity as evaluated by the use of $10 \mu \mathrm{mol} / \mathrm{l}$ AD P-induced platelet aggregation ( $p=0.002$ ).

The findings of our study investigating CYP2C 19 polymorphism in patients with coronary intervention for symptomatic CAD corroborate the assumption that patients carrying the CYP2C 19*2 allele are at risk for poor response to clopidogrel loading dose. Patients carrying at least one CYP2C19*2 allele had a fourfold increased risk $(p<0.0001)$ for developing high RPA. M oreover, the fact that the risk for developing high RPA increased by the presence of one CYP2C 19*2 allele (OR: 3.71) and was even more pronounced by two variant alleles (OR: 10.72) clearly reflects a gene- dose effect of clopidogrel action on ADP-mediated platelet aggregation. Why a recent study including a small number of cardiovascular outpatients did not show an impact of CYP2C 19 on clopidogrel responsiveness remains unclear [24].

Since clopidogrel requires bioactivation to its active metabolite 2-oxoclopidogrel via CYP2C 19 to irreversibly block the platelet AD P receptor $\mathrm{P}_{2} \mathrm{Y}_{12}$ [25], thereby resulting in an approximately $50 \%$ reduction in AD P-mediated platelet aggregation, the CYP2C 19*2 allele provides a plausible explanation for interindividual 


\begin{tabular}{|c|c|c|c|c|c|c|}
\hline \multirow{2}{*}{$\begin{array}{l}\text { CYP } \\
\text { polymorphism }\end{array}$} & \multicolumn{3}{|c|}{ Patients } & \multirow[t]{2}{*}{ Statistics } & \multicolumn{2}{|c|}{ Low vs high RPA } \\
\hline & Genotype & $\begin{array}{l}\text { Low RPA } \\
(\%)\end{array}$ & $\begin{array}{l}\text { High RPA } \\
(\%)\end{array}$ & & $\begin{array}{l}\chi^{2} \text { or odds ratio } \\
(95 \% \mathrm{Cl})\end{array}$ & p-value \\
\hline \multirow{3}{*}{$\begin{array}{l}\text { CYP2C19*2 } \\
(n=237)\end{array}$} & $* 1 / * 1$ & $144(82.3)$ & $31(50)$ & $* 1 / * 1$ vs $* 1 / * 2+* 2 / * 2$ & $4.6(2.5-8.7)$ & $<0.0001$ \\
\hline & $* 1 / * 2$ & $28(16)$ & $24(38.7)$ & $* 1 / * 1$ vs $* 1 / * 2$ vs $* 2 / * 2$ & 27.17 & $<0.001$ \\
\hline & $* 2 / * 2$ & $3(1.7)$ & $7(11.3)$ & - & - & - \\
\hline \multirow{3}{*}{$\begin{array}{l}\text { CYP2C19*17 } \\
(n=237)\end{array}$} & $* 1 / * 1$ & $96(54.9)$ & $41(66.1)$ & $* 1 / * 1$ vs $* 1 / * 17+* 17 / * 17$ & $0.62(0.34-1.14)$ & 0.14 \\
\hline & $* 1 / * 17$ & $65(37.1)$ & $14(22.6)$ & $* 1 / * 1$ vs $* 1 / * 17$ vs $* 17 / * 17$ & 4.48 & 0.11 \\
\hline & $* 17 / * 17$ & $14(8.0)$ & $7(11.3)$ & - & - & - \\
\hline \multirow{3}{*}{$\begin{array}{l}\text { CYP3A4*1B } \\
(n=234)\end{array}$} & $* 1 / * 1$ & $162(94.2)$ & $57(91.9)$ & $* 1 / * 1 \mathrm{vs} * 1 / * 1 \mathrm{~B}+* 1 \mathrm{~B} / * 1 \mathrm{~B}$ & $1.42(0.46-4.33)$ & 0.55 \\
\hline & $* 1 / * 1 \mathrm{~B}$ & $10(5.8)$ & $4(6.5)$ & $* 1 / * 1$ vs $* 1 / * 1 \mathrm{~B}$ vs $* 1 \mathrm{~B} / * 1 \mathrm{~B}$ & 2.83 & 0.24 \\
\hline & $* 1 \mathrm{~B} / * 1 \mathrm{~B}$ & $0(0)$ & $1(1.6)$ & - & - & - \\
\hline \multirow{3}{*}{$\begin{array}{l}\text { CYP3A5*3 } \\
(n=235)\end{array}$} & $* 3 / * 3$ & $149(86.1)$ & $53(85.5)$ & $* 3 / * 3$ vs $* 1 / * 3+* 1 / * 1$ & $1.05(0.46-2.41)$ & 1.00 \\
\hline & $* 1 / * 3$ & $24(13.9)$ & $8(12.9)$ & $* 3 / * 3$ vs $* 1 / * 3$ vs $* 1 / * 1$ & 2.82 & 0.24 \\
\hline & $* 1 / * 1$ & $0(0)$ & 1 (1.6) & - & - & - \\
\hline
\end{tabular}

Cl: Confidence interval; RPA: Residual platelet aggregation.

variability of drug response. CYP2C $19 * 2$ results in a truncated protein unable to bind to the heme moiety [26], consequently leading to a nonfunctional enzyme. In addition, the CYP2C 19*17 allele was recently reported, resulting in a putatively increased enzyme function of CYP2C $19[17,27]$, and possibly affects clopidogrel response in the opposite direction as reflected by low RPA. In the present study, we firstly investigated the contribution of the CYP2C19*17 variant on RPA, but found no significant association (Table 3).

It is well established that CYP2C19 pharmacogenetics is of clinical relevance. For instance, carriers of two nonfunctional CYP 2 C 19*2 alleles have a decrease in oral clearance of proton pump inhibitors, such as omeprazole and lansoprazole, by approximately $85 \%$, and several studies demonstrated that cure rates of Helicobacter pylori infection were lower in CYP2C 19 wild-type individuals compared with homozygous variant or heterozygous patients [28,29]. Thus, CYP2C 19 pharmacogenetically-guided dose adjustment has been suggested to improve the clinical efficacy of proton pump inhibitors. Interestingly the frequency of CYP2C19 variant individuals varies among different racial groups [26]. By contrast to white and black subjects, among whom homozygous carriers occur at fequencies of $2-4 \%$, in Asian subjects the frequency of mutant subjects is substantially higher $(\sim 14.0 \%)$, particularly in Japanese $(20 \%)$.
Therefore, the impact of the CYP2C19*2 polymorphism on clopidogrel reponsiveness is expected to be of particular interest in patients of Asian origin.

In addition to CYP2C 19, genetic variants in CYP3A enzymes (CYP3A4 and CYP3A5) may contribute to poor response rates of clopidogrel. Both CYP3A4 and CYP3A5 are polymorphic, but unlike CYP3A5 (CYP3A5*3) there are no CYP3A4 variant alleles described so far that appear at high allele frequencies with marked differences between populations and that may alter CYP3A4 function to a clinically relevant extent [30,31]. O nly the CYP3A4*1B variant was suggested to contribute to small changes in CYP3A4 protein function [32]. $\mathrm{H}$ owever, by far the best-characterized genetic polymorphism in CYP3A genes is the CYP $3 A 5 * 3$ variant, which results in alternative splicing of an mR N A species harboring a premature termination codon and subsequent nonsense-mediated decay of the mRNA [33]. Consequently, homozygous carriers of CYP $3 A 5 * 3$, who represent the vast majority of $C$ aucasians, express up to 30-fold lower levels of CYP3A5 protein compared with subjects, who carry at least one CYP3A5*1 wild-type allele [33,34]. Alteration of drug disposition for several agents (e.g., tacrolimus), which are CYP3A substrates has been described in the presence of CYP3A5 polymorphisms [35]. Interestingly, we did not find any contribution of both the CYP $3 A 4 * 1 B$ and CYP $3 A 5 * 3$ polymorphism on RPA in our 
Figure 2. Nomogram for estimating individual risk for high residual platelet aggregation under a loading dose of $600 \mathrm{mg}$ clopidogrel.

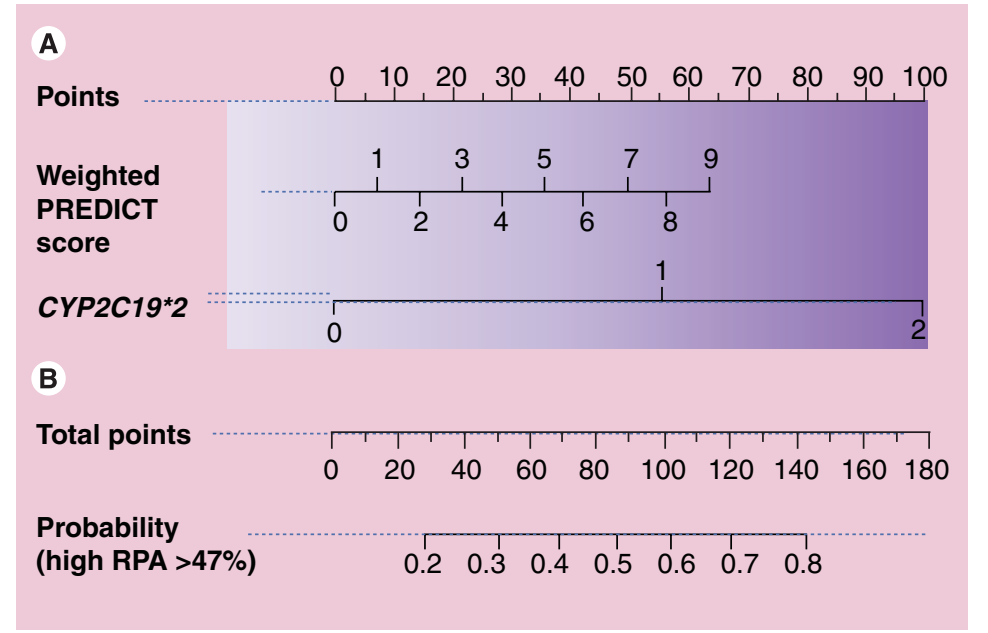

The nomogram was developed on the basis of the final logistic regression model (see statistical analysis). It allows calculating the probability for high RPA $(>47 \%$ ) based on nongenetic factors (weighted PREDICT score) and CYP2C19*2 genotype (0: CYP2C19 wild-type, 1: CYP2C19*1/*2,

2: CYP2C19*2/*2). For each factor on the left of panel (A) the appropriate number of points is determined on the upper scale. The total sum of points is transformed into the estimated probability panel (B) for a high RPA.

RPA: Residual platelet aggregation.
$\mathrm{PCI}$ [37]. In concordance with our data, CYP3A5 did not explain variability in clopidogrel response as found by those authors.

Taken together, whereas the evidence is conclusive that the CYP2C19*2 polymorphism is significantly associated with reduction of ADP-induced platelet aggregation (present study, $[9,20,21,23])$, the contribution of CYP3A4/5 to clopidogrel response remains open. Interestingly, it was shown that inhibition of CYP3A4/5 by ketoconazole, the most potent inhibitor of CYP3A, significantly affects formation of the active metabolite of clopidogrel in healthy volunteers [38]. Since ketoconazole also inhibits CYP2C 19 even if to a smaller extent, additional data are required.

The concept that inheritance can have an important role in individual variation of drug response is complemented by the fact that, additionally, nongenetic factors such as age, disease status, organ function, comedication and so on, may influence drug efficacy $[39,40]$. Recently, we demonstrated that five nongenetic weighted variables (PREDICT-score: age $>65$ years, Type 2 diabetes mellitus, decreased left ventricular function, renal failure and acute coronary syndrome) render patients more susceptible to high RPA, consequently resulting in an increased risk for poor response to clopidogrel treatment [8]. Thus, an important finding of the present study is the fact that our multivariable logistic regression analysis showed an even higher association with high RPA for the combination of nongenetic risk factors (PREDICT-score) and the CYP2C 19*2 genotype $\left(\chi^{2}=25.85 ; p<0.0001\right)$. These data indicate that clopidogrel nonresponsiveness depends on both genetic and nongenetic (i.e., physiological/pathophysiological) factors. Finally, we developed a nomogram (Figure 2) based on our dichotomous logistic regression model, which includes both genetic and nongenetic factors to individually estimate the probability that a given patient will be susceptible to high RPA after first treatment with a 600-mg clopidogrel loading dose. For each factor the appropriate number of points is determined and the total sum of points is transformed into the estimated probability (see results section). The nomogram underscores one of the important findings of our study, namely that genetic and nongenetic factors may contribute to poor responsiveness of clopidogrel loading dose. However, before translation of our nomogram into practical clinical use 
prospective clinical trials, including representative large patient cohorts, are warranted with particular interest in antithrombotic outcome under long-term clopidogrel therapy.

\section{Conclusion}

In summary, antithrombotic therapy in patients after coronary stenting is well established, but long-term major cardiovascular events occurred in up to $10 \%$ of patients, despite dual therapy with aspirin and clopidogrel (Clopidogrel for $\mathrm{H}$ igh Atherothrombotic Risk and Ischemic Stabilization, $\mathrm{M}$ anagement, and Avoidance [CH ARISMA] trial) [41]. There is substantial need, particularly in high-risk patients with vascular disease or multiple risk factors, to optimize antithrombotic therapy and to identify patients with poor responsiveness before initiating clopidogrel therapy. Thus, clopidogrel therapy tailored on the basis of pharmacogenetic knowledge, including the individual CYP2C19*2 genotype, but also considering nongenetic factors such as age over 65 years, Type 2 diabetes mellitus, decreased left ventricular function, renal failure and acute coronary syndrome, offers a novel dimension in antithrombotic therapy.

Financial \& competing interests disclosure The authors have no relevant affiliations or financial involve ment with any organization or entity with a financial interest in or financial conflict with the subject matter or materials discussed in the manuscript. This includes employment, consultancies, honoraria, stock ownership or options, expert testimony, grants or patents received or pending, or royalties

No writing assistance was utilized in the production of this manuscript.

\section{Ethical conduct of research}

The authors state that they have obtained appropriate institutional review board approval or have followed the principles outlined in the Declaration of $\mathrm{H}$ elsinki for all human or animal experimental investigations. In addition, for investigations involving human subjects, informed consent has been obtained from the participants involved.

\section{Executive summary}

- Clinical outcome in patients undergoing percutaneous coronary intervention is significantly improved if patients are pretreated with a loading dose of clopidogrel, an inhibitor of platelet aggregation.

- Due to a marked interindividual variability in clopidogrel-induced inhibition of platelet aggregation, up to $30 \%$ of all patients do not achieve a sufficient antiplatelet effect. In a recent large-scale study, nongenetic factors were identified for better prediction of patients with high residual platelet activity after coronary stenting and clopidogrel therapy.

- In the present study, we investigated the impact of functional polymorphisms in the drug-metabolizing enzymes CYP2C19 and CYP3A4/5 on clopidigrel responsiveness after a loading dose of $600 \mathrm{mg}$. Carriers of the CYP2C19*2 variant demonstrated significantly increased residual-platelet activation (RPA) compared with noncarriers.

- Multivariable logistic regression analyses showed that CYP2C19*2 genotype contributes predominately to high RPA in comparison with nongenetic risk factors. For the combination of both, an even higher association was found.

- These findings indicate that poor responsiveness after clopidogrel loading dose depends on both genetic and nongenetic factors and may be improved by genotyping for CYP2C19*2.

\section{Bibliography}

Papers of special note have been highlighted as either of interest $(\bullet)$ or of considerableinterest $(\cdot \bullet$ to readers.

1. Steinhubl SR, Berger PB, M ann JT et al.: Early and sustained dual oral antiplatelet therapy following percutaneous coronary intervention: a randomized controlled trial. JAM A 288(19), 2411-2420 (2002).

2. Kastrati $A, M$ ehilli J, Schuhlen $H$ et al.: A clinical trial of abciximab in elective percutaneous coronary intervention after pretreatment with clopidogrel. N. Engl. J. M ed. 350(3), 232-238 (2004).

3. Gurbel $P A, B l i d e n ~ K P, H$ iatt $B L$, $O$ 'Connor CM : Clopidogrel for coronary stenting: response variability, drug resistance, and the effect of pretreatment platelet reactivity. Circulation 107(23), 2908-2913 (2003).

4. Gurbel PA, Bliden KP: Platelet activation after stenting with heparin-coated versus noncoated stents. Am. H eart. J. 146(4), E10 (2003).

5. M uller I, Besta F, Schulz C, M assberg S, Schonig A, Gawaz M : Prevalence of clopidogrel non-responders among patients with stable angina pectoris scheduled for elective coronary stent placement. Thromb. H aemost. 89(5), 783-787 (2003).

6. M atetzky S, Shenkman B, Guetta $V$ et al.: Clopidogrel resistance is associated with increased risk of recurrent atherothrombotic events in patients with acute myocardial infarction. Circulation 109(25), 3171-3175 (2004).

7. Geisler $\mathrm{T}$, Langer $\mathrm{H}$, Wydymus $\mathrm{M}$ et al.: Low response to clopidogrel is associated with cardiovascular outcome after coronary stent implantation. Eur. H eart. J. 27(20), 2420-2425 (2006).

8. Geisler T, Grass D, Bigalke B et al.: The Residual Platelet Aggregation after D eployment of Intracoronary Stent (PRED ICT ) score. J. Thromb. H aemost. 6(1), 54-61 (2008).

- Recent report of a combination of nongenetic factors predicting clopidogrel poor response.

9. Hulot JS, Bura A, Villard E et al.: Cytochrome P450 2C 19 loss-of-function polymorphism is a major determinant of clopidogrel responsiveness in healthy subjects. Blood 108(7), 2244-2247 (2006).

- First report of an association between clopidogrel responsiveness and CYP2C19'2 polymorphism in healthy volunteers. 
10. Savi P, Pereillo JM , U zabiaga M F et al.: Identification and biological activity of the active metabolite of clopidogrel. Thromb. H aemost. 84(5), 891-896 (2000).

11. Lau WC, Gurbel PA: Antiplatelet drug resistance and drug-drug interactions: role of cytochrome P450 3A4. Pharm. Res. 23(12), 2691-2708 (2006).

12. Lau WC, Gurbel PA, Watkins PB et al.: Contribution of hepatic cytochrome P450 $3 \mathrm{~A} 4$ metabolic activity to the phenomenon of clopidogrel resistance. Circulation 109(2), 166-171 (2004).

13. Schaeffeler E, Eichelbaum M, Brinkmann U et al.: Frequency of C $3435 \mathrm{~T}$ polymorphism of M DR1 gene in African people. Lancet 358(9279), 383-384 (2001).

14. H ochholzer W, Trenk D, Frundi D et al.: Time dependence of platelet inhibition after a 600-mg loading dose of clopidogrel in a large, unselected cohort of candidates for percutaneous coronary intervention. Circulation 111(20), 2560-2564 (2005).

15. Gawaz M, Ruf A, N eumann FJ et al.: Effect of glycoprotein II b-III la receptor antagonism on platelet membrane glycoproteins after coronary stent placement. Thromb. H aemost. 80(6), 994-1001 (1998).

16. Gachet C: AD P receptors of platelets and their inhibition. Thromb. H aemost. 86, 222-232 (2001).

17. Schroth W, Antoniadou L, Fritz P et al.: Breast cancer treatment outcome with adjuvant tamoxifen relative to patient CYP2D 6 and CYP2C 19 genotypes. J. Clin. Oncol. 25(33), 5187-5193 (2007).

18. H arrell FE: Regression modeling strategies, with applications to linear models, survival analysis and logistic regression. Springer, NY, USA (2001).

19. H ochholzer W, Trenk D, Bestehorn H P et al.: I mpact of the degree of periinterventional platelet inhibition after loading with clopidogrel on early clinical outcome of elective coronary stent placement. J. Am. Coll. Cardiol. 48(9), 1742-1750 (2006).

20. Fontana $\mathrm{P}, \mathrm{H}$ ulot JS, D e M oerloose $\mathrm{P}$, Gaussem P: Influence of CYP2C 19 and CYP3A4 gene polymorphisms on clopidogrel responsiveness in healthy subjects. J. Thromb. H aemost. 5(10), 2153-2155 (2007).

21. Brandt JT, Close SL, Iturria SJ et al.: Common polymorphisms of $\mathrm{CYP} 2 \mathrm{C} 19$ and CYP2C 9 affect the pharmacokinetic and pharmacodynamic response to clopidogrel but not prasugrel. J. Thromb. H aemost. 5(12), 2429-2436 (2007).
- First report of pharmacokinetic alteration of clopidogrel metabolism by CYP2C 19 in healthy volunteers.

22. Brandt JT, Kirkwood S, M ukhopadhay N et al.: CYP2C 19*2 polymorphism contributes to a diminished pharmacodynamic responseto clopidogrel [abstract]. J. Am. Coll. Cardiol. 47, 380A (2006).

23. Giusti $B, G$ ori $A M, M$ arcucci $R$ et al.: Cytochrome P450 2C 19 loss-of-function polymorphism, but not CYP3A4 IVS10 + 12G/A and P2Y12 T744C polymorphisms, is associated with response variability to dual antiplatelet treatment in high-risk vascular patients. Pharmacogenet. Genomics 17(12), 1057-1064 (2007).

- First report of an association between clopidogrel responsiveness and CYP2C19 polymorphism in cardiovascular patients.

24. Fontana P, Senouf D, M ach F: Biological effect of increased maintenance dose of clopidogrel in cardiovascular outpatients and influence of the cytochrome P450 2 C 19*2 allele on clopidogrel responsiveness. Thromb. Res. 121(4), 463-468 (2008).

25. H ollopeter $G$, Jantzen $H M$, Vincent $D$ et al.: Identification of the platelet ADP receptor targeted by antithrombotic drugs. $N$ ature 409(6817), 202-207 (2001).

26. D esta Z, Zhao X, Shin JG, Flockhart D A Clinical significance of the cytochrome P450 2C 19 genetic polymorphism. Clin. Pharmacokinet. 41(12), 913-958 (2002).

27. Sim SC, Risinger $C, D$ ahl $M L$ et al.: A common novel CYP2C19 gene variant causes ultrarapid drug metabolism relevant for the drug response to proton pump inhibitors and antidepressants. Clin. Pharmacol. Ther. 79(1), 103-113 (2006).

28. Klotz U, Schwab M, Treiber G: CYP2C 19 polymorphism and proton pump inhibitors. Basic. Clin. Pharmacol. Toxicol. 95(1), 2-8 (2004).

29. Furuta $T$, Shirai $N$, Sugimoto $M, O$ hashi $K$, Ishizaki T: Pharmacogenomics of proton pump inhibitors. Pharmacogenomics 5(2), 181-202 (2004).

30. Schirmer M, Toliat M R, H aberl M et al.: $G$ enetic signature consistent with selection against the CYP3A4*1B allele in nonAfrican populations. Pharmacogenet. Genomics 16(1), 59-71 (2006).

31. Burk 0, Schwab $M:$ The limited impact of CYP3A5 genotype for the pharmacokinetics of CYP3A substrates. Eur. J. Clin. Pharmacol. 63(12), 1097-1098 (2007).

32. Wandel $C, W$ itte JS, $H$ all JM, Stein CM, Wood AJ, Wilkinson GR: CYP3A activity in African American and European American men: population differences and functional effect of the CYP3A4*1B 5 '-promoter region polymorphism. Clin. Pharmacol. Ther. 68(1), 82-91 (2000).

33. Kuehl $P$, Zhang J, Lin $Y$ et al.: Sequence diversity in CYP3A promoters and characterization of the genetic basis of polymorphic CYP3A5 expression. $N$ at. Genet. 27(4), 383-391 (2001).

34. Hustert $\mathrm{E}, \mathrm{H}$ aberl $\mathrm{M}$, Burk $\mathrm{O}$ et al.: The genetic determinants of the CYP $3 A 5$ polymorphism. Pharmacogenetics 11(9), 773-779 (2001).

35. Wojnowski L, Kamdem LK: Clinical implications of CYP3A polymorphisms. Expert. 0 pin. D rug. M etab. Toxicol. 2(2), 171-182 (2006).

36. Angiolillo DJ, Fernandez-O rtiz A, Bernardo $E$ et al.: Contribution of gene sequence variations of the hepatic cytochrome P450 3A4 enzyme to variability in individual responsiveness to clopidogrel. Arterioscler. Thromb. Vasc. Biol. 26(8), 1895-1900 (2006).

37. Smith $S M$, Judge $H M$, Peters $G$ et al.: Common sequence variations in the $\mathrm{P} 2 \mathrm{Y} 12$ and CYP $3 A 5$ genes do not explain the variability in the inhibitory effects of clopidogrel therapy. Platelets 17(4), 250-258 (2006).

38. Farid N A, Payne CD, Small DS et al.: Cytochrome P450 3A inhibition by ketoconazole affects prasugrel and clopidogrel pharmacokinetics and pharmacodynamics differently. Clin. Pharmacol. Ther. 81(5), 735-741 (2007).

39. Eichelbaum $M$, Ingelman-Sundberg $M$, Evans WE: Pharmacogenomics and individualized drug therapy. Annu. Rev. M ed. 57, 119-137 (2006).

40. Roden D M , Altman RB, Benowitz N L et al.: Pharmacogenomics: challenges and opportunities. Ann. Intern. M ed. 145(10), 749-757 (2006).

41. Bhatt D L, Fox KA, H acke W et al.: Clopidogrel and aspirin versus aspirin alone for the prevention of atherothrombotic events. N. Engl. J. M ed. 354(16), 1706-1717 (2006).

Websites

101. H ome Page of the H uman Cytochrome P450 (CYP) Allele N omenclature Committee www.cypalleles.ki.se/

102. The R Project for Statistical Computing www.r-project.org

103. $\mathrm{H}$ ardy-W einberg equilibrium http://ihg.gsf.de/cgi-bin/hw/hwal.pl 